\title{
Correspondence Method for Registration of Range Images Using Evolutionary Algorithms
}

\author{
John W. Branch ${ }^{1}$, Flavio Prieto ${ }^{2}$, Pierre Boulanger ${ }^{3}$
}

\begin{abstract}
Registration is a fundamental stage in the 3D reconstruction process, which is used to match two or more images that can be taken in different moments, from different sensors, or from different viewpoints. Genetic algorithms have been applied to the registration of images, because of their ability to solve problems of optimization. In this paper, a procedure is presented to solve the registration problem of multiple view range images using evolutionary algorithms. This procedure is focused, on the problem of obtaining the best correspondence between points through a robust method of search on images that are partially overlapped. This correspondence set will allow us to calculate a rigid transformation that registers the images accurately.
\end{abstract}

\section{INTRODUCTION}

$\mathrm{R}$ econstruction is the process whereby real objects are reproduced within computer memory. Physical characteristics such as dimension, volume and shape are represented in digital form. The task of surface reconstruction of $3 \mathrm{D}$ objects from range images covers several stages: acquisition, registration, integration, segmentation, and adjustment, which when are combined, transform a set of partial images of the object to a complete 3D model [5].

Registration is a fundamental stage in the 3D reconstruction process. It is used to match two or more images which can be taken at different times from different sensors, or from different views. The misalignment that is unavoidably produced when two or more images have been taken from different views, and without any control of the relative positions of the sensor and the object, becomes the central problem of registration.

The purpose of the registration process is to align these views in such a way that the object's shape is recovered with the highest precision. During this process two situations become evident. First, it is not possible to determine which of the coordinate system points of one image matches with the correspondence points of another image; this is known as the matching problem, and is the most time-consuming stage during the execution of the algorithm. Second, a transformation is required in the threedimensional information of one of the images regarding its coordinate system and its relationship to the image that was chosen as its reference.

1. John W. Branch. Prof. Escuela de Sistemas, Universidad Nacional de Colombia, Sede Medellín; e-mail: jwbranch@unalmed.edu.co.

2. Flavio Prieto. Prof, Dept. Eléctrica, Electrónica y Computación, Universidad Nacional de Colombia, Sede Manizales; e-mail: fprieto@nevado.manizales.unal.edu.co.

3. Pierre Boulanger. Prof. Department of Computing Science, University of Alberta Edmonton, Alberta, Canada; e-mail: pierreb@cs.ualberta.ca.
The purpose here is of adjusting both images using common information between them. Because of the inexact nature of the data, and the uncertainness of the common surfaces, the procedure to calculate this transformation is iterative and it is guided by the strategies and metrics that have been chosen by the researcher. Due to this, the registration process is one of the slowest and most delicate stages in the process of 3D reconstruction. The quality of the alignment process determines the quality of the model that will be obtained.

Since 1992, with the introduction of the ICP Algorithm [1] there have been many variations to mitigate its deficiencies. This algorithm formulated a basic schema to obtain the alignment while minimizing the cost function and is based on the squares summation of the distance between points on the image. The basic procedure involves features identification, matching of correspondent features, and the alignment of these matches, by evaluating a distance [1] [3]. This method is also composed of two basic procedures. The first one is to find matching points, and the second one is to estimate the transformations iteratively for these points until some stop distance criteria is satisfied.

Another approach to the registration of images consists in determining a set of matches through a search process instead of the classical approach based on distances. This approach consists in finding a solution close to the global minimum in a reasonable time. This can be done by means of a Genetic Algorithm (GA). Genetic Algorithms have been applied to the registration problem. These algorithms are computational methods based on the concepts of natural evolution. Evolution occurs through successive cycles of assignment, reproduction, mutation, and replacement until the desired solution is found [2].

In this paper, we show a procedure based on Genetic Algorithms for the registration problem in several range images. This procedure focuses on the problem of obtaining the best match between points through a robust search method on images that are partially overlapped. This set of matches allows the calculation of transformation which precisely registers the images.

This paper is organized as follows: Section 2 presents a literature review. Section 3 exposes the ICP algorithm. Section 4 describes the methodology used to do the matching of the range images using Genetic Algorithms. Section 5 presents experiments and an analysis of the results obtained, and in the Section 6, the conclusions of this work are presented.

\section{LITERATURE REVIEW}

In the last decade, there have been several algorithms proposed for registration. These can be classified as coarse and fine. 
The best known iterative methods for fine registration are the variants of the Iteration Closest Point Algorithm (ICP). ICP is an iterative process which with each iteration, minimizes the mean square error calculated as the summation of the distances between points on a view and the closest corresponding points on the other view. This method was proposed by Besl and McKay [1]. This is the classical approach for solving the registration problem.

However, the disadvantage of ICP is that it requires a good pre-alignment of the views so that it converges to an accurate solution. Many variants of ICP have been proposed to address these limitations. For the registration with regions that partially overlap, heuristics have been proposed to ignore the regions not overlapped and consequently to obtain more effective transformation. The main difference among these methods are in the evaluation functions which measure the quality of the alignments at every iteration, and a reject in rules, discard of points at the boundary; which could arrive at false matchings. Chen and Medioni [3] developed a mechanism which minimizes the summation of the square distances between points on a view in reference to a tangent plane on another view. This approach is faster than traditional ICP and its results are better if a good prealignment is provided. However, this approach presents some numerical drawbacks, because some matches cannot be found. Zhang [9] proposed a set of sophisticated modifications to ICP. This method automatically calculates a threshold which is used to classify a point as a result if its distance from a corresponding point exceeds this threshold. Masuda and Yokoya [4] proposed a robust method for the registration of a couple of dense range images which integrate ICP, random registration, and the estimator of the least square median. They also proposed a modification to the K-d tree to improve the point correspondence search and to accelerate the method.

Another approach to register two range images is to find the geometric transformation through a search space, rather than the searches based on matches using ICP-based methods. In this case, the goal is to find within a search space of geometrical transformations, a solution that can be employed to accurately align two views. A reasonable way to develop this search is through the use of efficient stochastic optimization techniques, such as Genetic Algorithms.

Brunnstrom and Stoddart [2] proposed a method that integrates the classical ICP method with a genetic algorithm to couple free form surfaces. Here an alignment is obtained with a genetic algorithm, which is later refined with the ICP. The main problem treated by Brunnstrom and Stoddart is to find a corresponding set of points between the two views. Robertson and Fisher [6] proposed a parallel genetic algorithm which reduces the computational time, but its solution is not more accurate than the ones obtained with the first method. Silva et al [8] proposed a method for the registration of range images, making two key contributions: The hybridization of a genetic algorithm with the heuristic optimization method of hill climbing, and a measurement of the performance of the interpretation of the surfaces different to the classical metric, based on the calculation of the mean square error between corresponding points on the two images after the registration.

Review of the literature about the registration problem reveals many attempts to solve it. The ICP algorithm has a distinguished place in these trials even though it has its limitations. For instance, although there is satisfactory convergence of ICP it can be guaranteed when one of the images is a subset of the other. When this condition is not fulfilled, erroneous alignments can be obtained. Although ICP is efficient, with a complexity average of $O(n \log n)$ where $n$ is the number of points on the image, the algorithm converges monotonically to a local minimum. Another disadvantage of ICP is that it requires a good pre-alignment of the views to converge to a correct solution. Some variants of ICP have been proposed to address these limitations.

It is difficult to compare the different proposals. Every researcher uses a different image base, which makes it very difficult to compare results due to the different metrics each employs. Sometimes, the strategy to pre-align the images can guide the process to a convergence which obtains an erroneous solution. As well, the strategies that exhaustively explore the space correspondences and transformations are computationally expensive. Although a reject may be made of the erroneous couples, this is not an adequate parameter to guarantee an adequate adjustment.

Evaluation of the accuracy of the adjustment is another item that requires attention. It is the easiest way to compare the obtained model with another model, synthetic or real.

Another topic that affects the performance of the method is the images' size. Modern scanners can offer elevated resolutions because the density of information of the images is high. Because of this, many sub-scanning strategies are proposed to reduce the number of corresponding points to guide the registration, the uniform random scanning, and the uniform scanning of the normals [7]. Another consideration in the registration problem are the rejection rules. These are strategies to depurate the matches, discard in the ones that are incorrect. One of the main rules is the exclusion of points at the boundaries. Its application is inexpensive and excluded regions are not overlapped. The parametric method offers advantages related to convergence speed and minimization to reach superior levels of accuracy. Therefore, it must be considered that the combination of parametric-minimization methods form an acceptable registratron.

\section{CORRESPONDENCE METHOD OF RANGE IMAGE POINTS USING A GENETIC ALGORITHM}

The general principle of a genetic algorithm is to subject a population of individuals to an evolutionary process, encoded as chromosomes, which represent some possible solutions to a searching problem. During evolution, an aptitude value is assigned to each individual obtained from a specifically defined function for the problem to be solved. This function, called aptitude function, should be designed in such a way that it favors the most apt or adequate 
individuals as the solution to the problem. The aptitude assigned to each individual is taken into account in the selection of the parents who will take part in the reproduction process. Here there is an exchange of genetic material or content of a pair of selected individuals to generate two new individuals or two new possible solutions to the problem that, according to a replacement mechanism, are incorporated into the population. The new descended individuals are also subjected to a mutation process which is a random perturbation of its genetic material in order to offer variability and also to enrich the exploration of the possible solutions to the problem. These are represented as chromosomes. Finally, after having completed a certain number of cycles of aptitude assignation, reproduction, mutation, and replacement (called generations), the individual with better aptitude is chosen as the best solution to the problem.

Genetic algorithms have been applied to the registration problem; however, the complexity of the search space has become a major challenge. A proposal to use genetic algorithms in the registration problem of the view of prealigned range images is presented and described. This proposal is based on searching a set of points that when taken as entrance to Horn's method, a very good transformation is obtained that allows the integration of images with a very small margin of error. Figure 1, shows a general diagram of the proposed method.

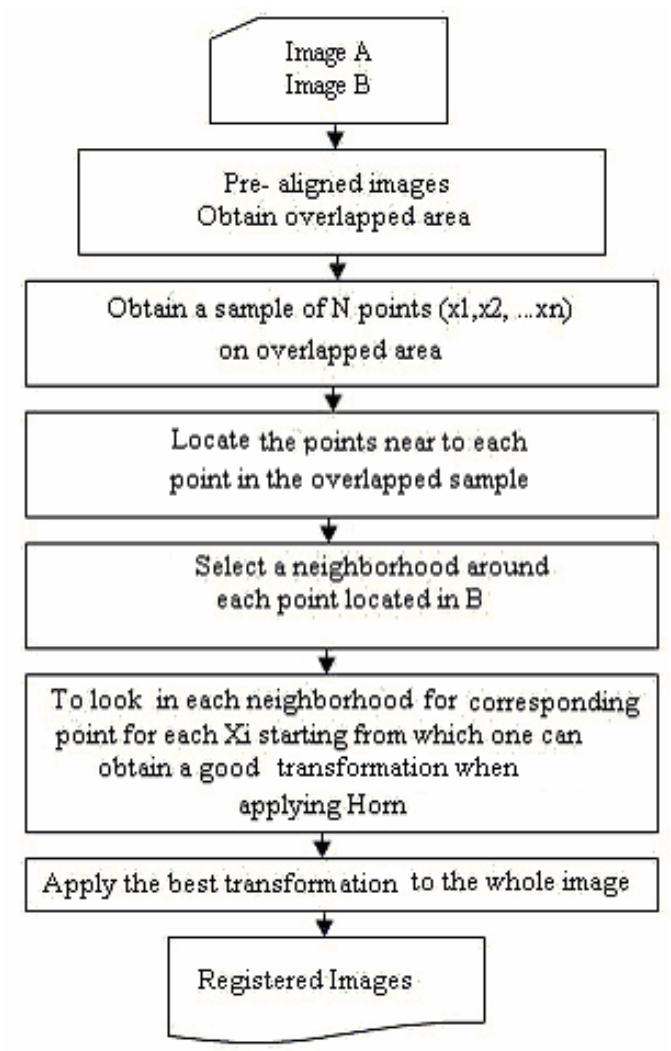

Fig. 1. General diagram of the proposed method.

The views to be registered are pre-aligned in order to obtain an initial overlapping area in both images. As it can be seen, in the following steps for each point of sample size $\mathrm{N}$ taken in the overlapping area of one of the views, a correspondent point is searched around the nearest points of the other view to be registered. This search is done because the best couple of points to obtain a transformation using Horn's method are not always the points with less distance within an overlapping area. Two views could be badly aligned and present points with very short distances; however when joining the views using these points as a guide, their registration could be off. The initially prealigned images could be askew and the correspondent points with which the views would match best when applying a transformation, could be very close to the points with a minimum distance.

Given two images of ranges $A$ and $B$ where $A$ is the image model and $\mathrm{B}$ is the image to be registered, searching the best points in A that match with a sample of points selected in $\mathrm{B}$, is done by a genetic algorithm. The design is as follows.

\section{A. Sampling}

It is a random selection of $\mathrm{N}$ points that belong to the overlapped area in B and establish, for each one of them, a subset of points or sub-domain in A. The subdomains contain $\mathrm{m}$ points near the closest point in A for each point in B. This approach of sub-domains reduces the search space and betters the global efficiency of the algorithm. The establishment of the domains has a critical computational step; that is, searching the closest point in A to each one of the points of the selected sample in B because this implies both calculating and comparing the distances to all the points which make up the overlapping area in A. Such a search is improved by implementing a K-d tree structure. Figure 2 graphically shows the establishment of a subdomain.

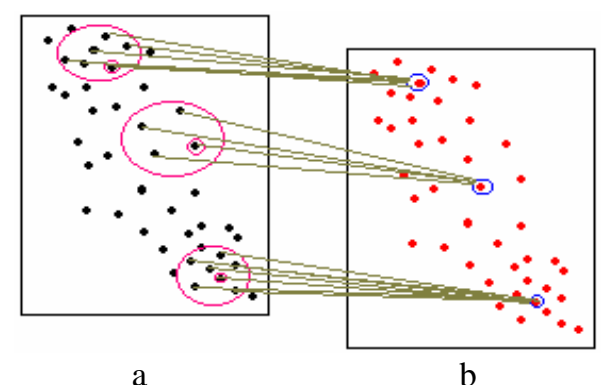

Fig. 2. Establishment of sub-domains, a) view A, b) view B

\section{B. Diagram of Representation}

Is represented as a chromosome of size $\mathrm{N}$, that is, to each one of the points of the selected sample in view $B$ there is a corresponding gene of the chromosome. Each gene contains an index that identifies a point within the neighborhood corresponding to a point, defined in view A. Figure 3 illustrates this representation.

\begin{tabular}{|l|l|l|l|l|}
\hline 12 & 25 & 78 & $\ldots \ldots$ & 1 \\
\hline 1 & 2 & 3 & $\mathrm{~N}$
\end{tabular}

Fig. 3. Diagram of representation of a chromosome.

Gene 1 corresponds to the first point of the sample, whereas gene 2 corresponds to the second point of the sample and subsequently to the $\mathrm{N}$-th point of the sample taken in view B. For instance, in Figure 3 gene 1 contains value 12, which means that point 12 is found within the subdomain corresponding to the first point of the sample in 
$\mathrm{B}$, twenty five (25) is an index of a point from view A that belongs to a neighborhood of points close to point 2 of the sample taken in view B. Each point of the sample taken in view $B$ has a defined neigborhood of points in view A from which the respective gene will take values.

\section{Aptitude function}

The aptitude function measures the average error between the points of the overlapping areas originating in the registration of the views. Each individual can be seen as a set of points with their respective couples translated into a transformation by Horn's method. The transformation is applied to the two views and the average error of this registration is assigned as the aptitude of an individual. The more accurate the individual, the smaller the error:

$$
\varepsilon=\sqrt{\frac{\sum_{i=1}^{N}\left(P_{i}-R_{i}\right)^{2}}{N}}
$$

$\mathrm{P}$ denotes each point in the overlapping area in view A obtained by applying each transformation.

$\mathrm{R}$ is each point in the overlapping area in view $\mathrm{B}$ after applying the transformation.

\section{Genetic operators}

The proposal presented for a two-view registration applies a simple cross with only one cut point, in which the parents' genetic content is exchanged on each side of the cut point in order to generate two new individuals (see Figure 4). In turn, the mutation operator varies the information of each gene according to the mutation probability, taking into account the defined neighborhoods for each point represented. That is, if gene i represents the i-th value of the sample taken in view $B$, and it has to be mutated, a respective point in the defined neighborhood is selected at random in view $\mathrm{A}$, and it is changed by the former value.

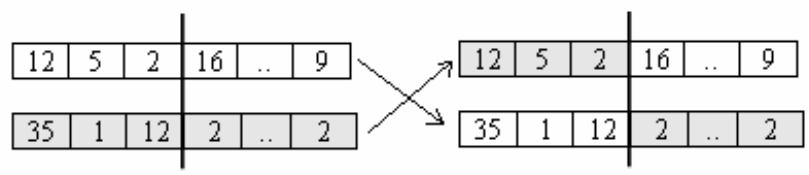

Fig. 4. Crossing with only one cut point.

\section{EXPERIMENTS AND RESULTS}

\section{A. Setting up the Experiment}

\section{1) Hardware and Libraries}

All tests were performed using a computer with a 3.0G processor and a 1.0G RAM memory, running under a Microsoft XP operating system. Model implementation was performed on $\mathrm{C}++$ and a graphic motor was programmed on Open GL in order to obtain the graphic representation of images. The data used were obtained with a Kreon sensor in the Advanced Man-Machine Interface Laboratory, Department of Computing Science, University of Alberta, Canada.

\section{2) Parametering of the Genetic Algorithm (GA)}

The execution of a GA, demands assigning proper values to a set of parameters that guarantee good performance. Within such a set of parameters we can find, among others, the population size, the individuals' length, and the probabilities assigned to each one of the genetic operators.

In order to establish the adequate values of the probabilities of the genetic operators for the problem of registration of the range images, a dozen pair of real range images were used with a variation of the probabilities of the crossing and mutation operators in intervals of $10 \%$. The results were obtained for each one of the possible combinations of their percentages. Table 1, shows the average of the results for each combination after 100 generations of the GA evolution.

The results with less average were obtained with a $60 \%$ probability for the crossing operator and $70 \%$ for the mutation operator. The size of the population was established at 100 individuals, each one of which is formed by 10 pairs of points. Table 2, shows the parameter values of the set up for the GA used in the different experiments performed in this work.

\section{3) Calibration of the Models}

Due to the fact that the GA model works on a specific problem, finding the best relationship between points that allows a transformation that correctly registers a pair of images with the objective of validating the correct performance of the methods ICP and ICP+GA, tests were performed to assure a point-to-point correspondence between the images, guaranteeing the existence of a unique solution to the problem.

The experiment consisted of the selection of a range image from a scanned real object that was used as a model image for the registration process. The registered image was generated by a variation of the points of a copy of the model image with a matrix of a known transformation. This generated a pair of images in which the existence of a unique point to point correspondence is guaranteed.

This experiment was performed with a GA that evolved through100 generations whose parameters were defined by the test performed in the former section. The convergence value of the GA was established at $1 \times 10^{-10}$. This is a very low value to find the best possible solution during the totality of the generations. The transformation matrix used to generate the image to be registered was built in three different cases. In the first case, the image was changed in each one of the rotation angles. In the second case, the transfer parameters were changed, and finally, a matrix was generated with the combination of the two former cases (see Figures 5, 6, and 7).

The results of these experiments showed that the ICP and ICP+GA methods are capable of finding a solution and allowing the registration of the pair of images given in each one of the different cases. The error in the images final registration using both methods shows a convergence to the global minimum (see Table 3, Table 4 and Table 5). Convergence was reached after 35 iterations or generations, demonstrating very fast convergence towards a solution.

\section{B. Analysis of results}

In this section, we analyze both the results obtained by the ICP method and a variant based on orientation of the normal with the proposed method of GA, using a real application. The object registered is a mask, composed of 8 range images obtained using the Kreon sensor. 
The process of registration was performed by registering each image by fusing it with images previously registered. From Figure 8 until Figure 15 is shown the behavior of the adjustment error and the sequence of the registration process. The final registered object is shown in the Figure 15.

The graphic results correspond to those obtained with the model GA through 100 generations. As it can be seen in Figure 16, in all cases a minor registration error was obtained with the model GA (Average Error = 0,0260), compared to the classical model ICP (Average Error =
0,0394), and the variant ICP+ Normal (Average Error = 0,0308). Each model was executed with the same number of iterations or generations and the values for the parameters of the GA model were established by the probes performed in the former sections. However, the time differences between the different methods and the GA are meaningful for these probes in which the images contain an average of 35,000 points. The classical methods take an average of 1.5 minutes to register each pair of views, whereas the GA model takes an average of 3.5 minutes for each pair of views.

TABLE 1

AVERAGE OF THE RESULTS OBTAINED FOR EACH COMBINATION OF THE PERCENTAGES ASSIGNED TO THE GENETIC OPERATORS

\begin{tabular}{|c|c|c|c|c|c|c|c|c|c|c|c|}
\hline \multirow[b]{3}{*}{ M } & \multicolumn{11}{|c|}{ Crossover } \\
\hline & & 0.1 & 0.2 & 0.3 & 0.4 & 0.5 & 0.6 & 0.7 & 0.8 & 0.9 & 1.0 \\
\hline & 0.1 & 0.0356 & 0.0488 & 0.0598 & 0.0413 & 0.0561 & 0.0594 & 0.0496 & 0.0473 & 0.0585 & 0.0473 \\
\hline \multirow{9}{*}{$\begin{array}{l}\mathbf{u} \\
\mathbf{t} \\
\mathbf{a} \\
\mathbf{t} \\
\mathbf{i} \\
\mathbf{0} \\
\mathbf{n}\end{array}$} & 0.2 & 0.0531 & 0.0498 & 0.0555 & 0.0259 & 0.0464 & 0.0358 & 0.0448 & 0.0377 & 0.0425 & 0.0506 \\
\hline & 0.3 & 0.0650 & 0.0597 & 0.0479 & 0.0447 & 0.0485 & 0.0521 & 0.0448 & 0.0499 & 0.0603 & 0.0376 \\
\hline & 0.4 & 0.0461 & 0.0382 & 0.0497 & 0.0593 & 0.0577 & 0.0531 & 0.0386 & 0.0311 & 0.0507 & 0.0358 \\
\hline & 0.5 & 0.0511 & 0.0501 & 0.0550 & 0.0518 & 0.0415 & 0.0479 & 0.0506 & 0.0554 & 0.0408 & 0.0373 \\
\hline & 0.6 & 0.0577 & 0.0512 & 0.0473 & 0.0267 & 0.0605 & 0.0504 & 0.0545 & 0.0542 & 0.0337 & 0.0425 \\
\hline & 0.7 & 0.0546 & 0.0446 & 0.0511 & 0.0455 & 0.0258 & 0.0208 & 0.0568 & 0.0463 & 0.0211 & 0.0350 \\
\hline & 0.8 & 0.0613 & 0.0490 & 0.0568 & 0.0569 & 0.0494 & 0.0521 & 0.0350 & 0.0542 & 0.0434 & 0.0423 \\
\hline & 0.9 & 0.0425 & 0.0512 & 0.0635 & 0.0470 & 0.0460 & 0.0486 & 0.0477 & 0.0458 & 0.0394 & 0.0551 \\
\hline & 1.0 & 0.0534 & 0.0480 & 0.0587 & 0.0402 & 0.0476 & 0.0509 & 0.0390 & 0.0512 & 0.0430 & 0.0654 \\
\hline
\end{tabular}

TABLE 2

VALUES OF THE GA'S SET UP

\begin{tabular}{|c|c|}
\hline PARAMETER & VALUE \\
\hline Number of generations & 100 \\
\hline Size of population & 100 \\
\hline Size of chromosome & 10 Pairs of gene \\
\hline Crossing probability & $60 \%$ \\
\hline Mutation probability & $70 \%$ \\
\hline
\end{tabular}

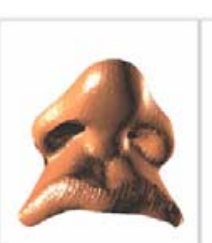

(a)

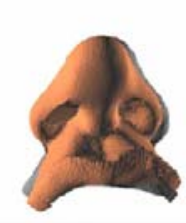

(b)

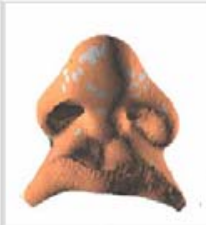

(c)
Fig. 5. Case 1: Rotation of the synthetic data, a) model image,

b) model image and image to be registered, c) registered images.

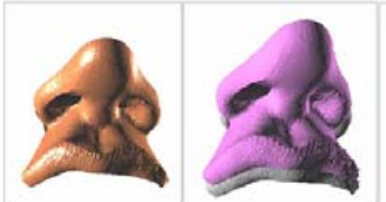

(a)

(b)

Fig. 6. Case 2: Transfer of the synthetic data, a) model image, b) model image and image to be registered, c) registered images.

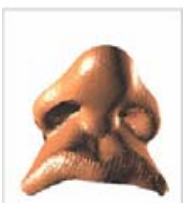

(a)

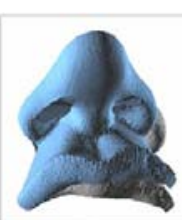

(b)

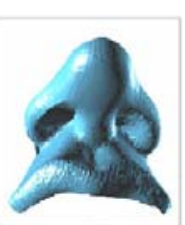

(c)
Fig.7. Case 3: Rotation and transfer of the synthetic data, a) model image, b) model image and image to be registered, c) registered images.

TABLE 3

OBTAINED TRANSFORMATIONS OF CASE 1 BY MEANS OF ICP AND THE MODEL AG

\begin{tabular}{|c|c|c|c|c|c|c|c|c|}
\hline CASE 1 & E & Rx & Ry & Rz & Tx & Ty & Tz & 0 \\
\hline Transformation & 1 & 0.070 & 0.05 & 0.03 & 0 & 0 \\
\hline ICP & 0.999 & 0.070 & 0.049 & 0.029 & 0.157 & 0.026 & 0.048 & $5.22 \mathrm{E}-10$ \\
\hline ICP+AG & 0.999 & 0.069 & 0.048 & 0.031 & $2.31 \mathrm{E}-18$ & $1.5 \mathrm{E}-226$ & $6.32 \mathrm{E}-15$ & $4.31 \mathrm{E}-11$ \\
\hline
\end{tabular}

TABLE 4

OBTAINED TRANSFORMATIONS OF CASE 2 BY MEANS OF ICP AND THE MODEL AG

\begin{tabular}{|c|c|c|c|c|c|c|c|c|}
\hline CASE 2 & E & Rx & Ry & Rz & Tx & Ty & Tz & Error \\
\hline Transformation & 1 & 0 & 0 & 0 & 1 & 3 & 1 \\
\hline ICP & 0.999 & 0.0378 & 0.03573 & 0.0261 & 1.089 & 3.204 & 1.2635 & $5.78 \mathrm{E}-11$ \\
\hline ICP+AG & 0.999 & $5.31 \mathrm{E}-48$ & $6.2 \mathrm{E}-22$ & $5.82 \mathrm{E}-15$ & 0.9865 & 3.0246 & 0.9856 & $4.29 \mathrm{E}-12$ \\
\hline
\end{tabular}

TABLE 5

OBTAINED TRANSFORMATIONS OF CASE 3 BY MEANS OF ICP AND THE MODEL AG.

\begin{tabular}{|c|c|c|c|c|c|c|c|c|}
\hline CASE 3 & $\mathbf{E}$ & $\mathbf{R x}$ & Ry & $\mathbf{R z}$ & $\mathbf{T x}$ & Ty & $\mathbf{T z}$ & Error \\
\hline Transformation & 1 & 0.070 & 0.05 & 0.03 & 1 & 3 & 1 & \\
\hline ICP & 0.999 & 0.067 & 0.058 & 0.043 & 1.251 & 2.974 & 1.524 & $6.52 \mathrm{E}-10$ \\
\hline ICP+AG & 0.999 & 0.069 & 0.055 & 0.027 & 1.025 & 3.036 & 0.856 & $3.52 \mathrm{E}-12$ \\
\hline
\end{tabular}




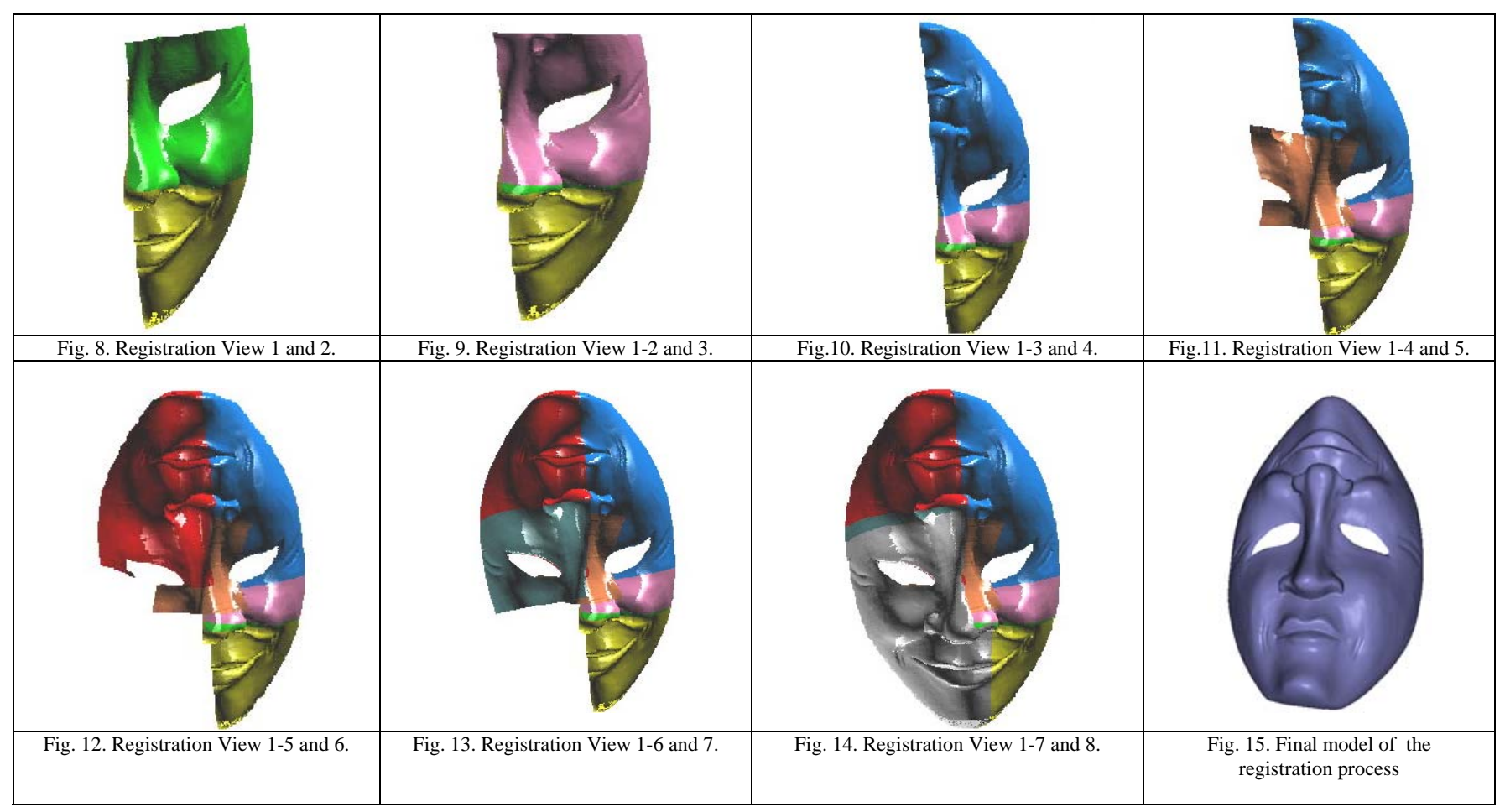

\begin{tabular}{|c|c|c|c|}
\hline & ICP & ICP+Norm & ICP+GA \\
\hline Pair 1 & 0,0355 & 0,0317 & 0,0295 \\
\hline Pair 2 & 0,0349 & 0,0314 & 0,0288 \\
\hline Pair 3 & 0,0181 & 0,0175 & 0,0169 \\
\hline Pair 4 & 0,0493 & 0,0338 & 0,0274 \\
\hline Pair 5 & 0,0360 & 0,0293 & 0,0197 \\
\hline Pair 6 & 0,0516 & 0,0361 & 0,0296 \\
\hline Pair 7 & 0,0501 & 0,0356 & 0,0298 \\
\hline
\end{tabular}

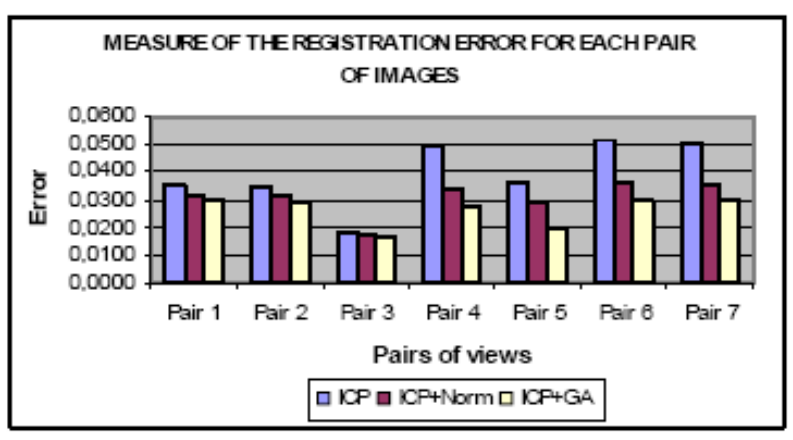

Fig. 16. Registration error for each pair of images

\section{CONCLUSIONS AND FUTURE WORK}

A semiautomatic method has been proposed for the registration of multiple view range images with low overlap that is capable of finding an adequate registration without needing a fine preliminary pre-alignment of the images. This method is based on a genetic algorithm to perform a query of the best correspondence between a set of sample points, starting from an approach based on sub-domains that reduces the search space of the genetic algorithm which implies global algorithm efficiency.

The comparison of the results obtained through the different experiments shows a more precise convergence (using proposed method (ICP+GA)) than the classical ICP method and one of its variants (ICP+ Normals) can provide. However, the proposed method takes more computational time to find the solution.

A diagram for the reconstruction of the 3D model allows for obtaining partial models, where the final result can be presented as combinations of triangular meshes or a cloud of points and the final error is defined as the average of the partial errors obtained in the registration of each pair of views.
For future work, the exploration of a parallel version to reduce the computational cost of the proposed method is suggested.

\section{REFERENCES}

[1] P. Besl. A method for registration of 3-d shapes. IEEE Trans. Pattern Anal. Mach. Intell; 14, 1992.

[2] K. Brunnstrom. Genetic algorithms for free-form surface matching. 1996.

[3] Y. Chen. Object modeling by registration of multiple range images. Image and Vision Computing, 10, 1992.

[4] T. Masuda. A robust method for registration and segmentation of multiple range images. Computer Vision and Image Understanding, 61(3), 1995.

[5] A. Myers. Introductory literature review surface reconstruction from three dimensional range data. Technical report, The University of Adelaidey, Department of Computer Science, 1999.

[6] C. Robertson. Parallel evolutionary registration of range data. Computer Vision and Image Understanding, 87, 2002.

[7] S. Rusinkiewiczs. Real-time Acquisition and Rendering of Large 3D Models. PhD thesis, Stanford University, 2001.

[8] L. Silva. Precision range image registration using a robust surface interpretation measure an enhanced genetic algorithm. IEEE Trans. Pattern Analysis and Machine Intelligence, 27(05), 2005.

[9] Z. Zhang. Iterative point matching for registration of free-form curves and surfaces. International Journal of Computer Vision, 13(2), 1994. 